SU-4252-864

\title{
Metastable Kinks in the Orbifold
}

\author{
Manuel Toharia and Mark Trodden \\ Department of Physics, Syracuse University, Syracuse, NY 13244, USA
}

(Dated: November 1, 2018)

\begin{abstract}
We consider static configurations of bulk scalar fields in extra dimensional models in which the fifth dimension is an $S^{1} / Z_{2}$ orbifold. There may exist a finite number of such configurations, with total number depending on the size of the orbifold interval. We perform a detailed Sturm-Liouville stability analysis that demonstrates that all but the lowest-lying configurations - those with no nodes in the interval - are unstable. We also present a powerful general criterion with which to determine which of these nodeless solutions are stable. The detailed analysis underlying the results presented in this letter, and applications to specific models, are presented in a comprehensive companion paper [1].

PACS numbers:
\end{abstract}

The possibility of extra spatial dimensions $2,3,3,4,5,6$, 7, 8, 9], hidden from our current experiments and observations through compactification or warping, has opened up a wealth of options for particle physics model building and allowed entirely new approaches for addressing cosmological problems.

In many models, standard model fields are supposed to be confined to a submanifold, or brane, while in other models they populate the entire bulk. Common to both approaches, however, is the inclusion of bulk fields beyond pure gravity, either because they are demanded by a more complete theory, such as string theory, or because they are necessary to stabilize the extra dimensional manifold. The allowed configurations of such bulk fields are determined, naturally, by their equations of motion, subject to the boundary conditions imposed by the particular extra-dimensional model under consideration. These might be periodic boundary conditions, in the case of a smooth manifold, or reflection-symmetric ones in the case of an orbifolded extra dimension.

In this letter we study a class of allowed nontrivial scalar field configurations 10, 11, 12, 13 in orbifolded extra-dimensional models, neglecting gravity. These configurations exist whenever the potential possesses at least two degenerate minima and we show that they may form a finite tower of kink state solutions. We explicitly demonstrate that all but the lowest-lying of these the ones with no nodes in the interval - are unstable. In addition we identify a stability criterion for these lowestlying states, and provide concrete examples for specific convenient choices of potential.

A complete understanding of the predictions and allowed phenomenology of extra dimension models necessarily includes a comprehensive consideration of the configurations of bulk fields. That a finite tower of nontrivial static configurations may exist, with the possibility of multiple stable ones, allows for new phenomena and con- straints on the models, and may have wide-ranging implications for the particle physics and cosmological theories constructed around them.

We are currently performing a much more detailed analysis of these configurations, including gravitational effects.

The model we consider consists of a real scalar field in 5 dimensions (labeled by indices $M, N, \ldots=0,1,2,3,5$ ) around a flat background metric, and defined by the action

$$
S=\int d^{5} x\left[\frac{1}{2} \eta^{M N} \partial_{M} \phi(x, y) \partial_{N} \phi(x, y)-V(\phi)\right],
$$

in which $\phi$ has units of (Mass) $)^{3 / 2}$. The extra dimension is compactified on an orbifold $S_{1} / Z_{2}$ with the scalar field $\phi(x, y)$ being odd under $Z_{2}$ reflections along the extra coordinate $x^{5} \equiv y$ (i.e. $\phi(x, y)=-\phi(x,-y)$ ). Here the orbifold interval is defined as $[0, \pi R]$, with its size $\pi R$ assumed to be fixed.

The potential $V(\phi)$ must then be invariant under the discrete symmetry $\phi \rightarrow-\phi$, and is chosen to have two degenerate global minima at $\phi= \pm v$ with $v \neq 0$. To simplify notation, we will also choose the potential to vanish at $\phi=0$.

We seek static field configurations $\phi_{A}(y)$, parametrized by their amplitudes $A$, which extremize the action, and with nontrivial $y$-dependence, subject to the appropriate boundary conditions, namely $\phi_{A}(0)=0$ and $\phi_{A}(\pi R)=0$. The field equation satisfied by such solutions is

$$
\phi_{A}^{\prime \prime}-\frac{\partial V}{\partial \phi_{A}}=0,
$$

where a prime denotes a derivative with respect to $y$. It is easily seen that there exists a first integral, given by

$$
\frac{1}{2} \phi_{A}^{\prime 2}-V\left(\phi_{A}\right)=E_{A} \text {. }
$$


To better understand the problem, it is extremely helpful to note (Figure 11) that (2) and (3) are precisely the equations of a particle rolling in time $(y)$, without friction, in the inverted potential $U(\phi) \equiv-V(\phi)$.

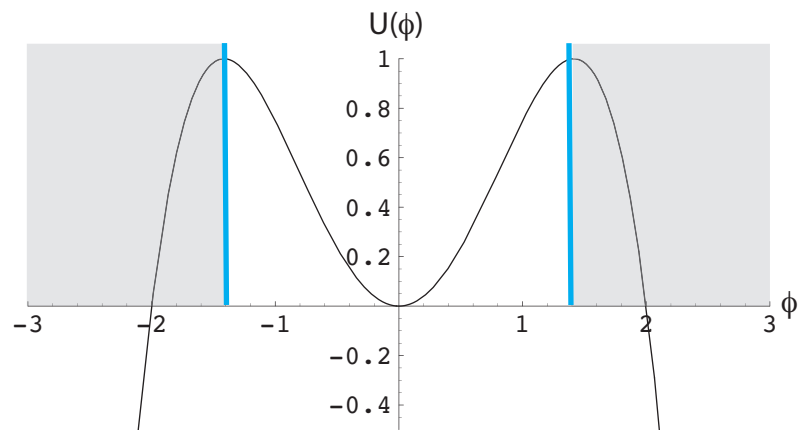

FIG. 1: Mechanical Analogy: Periodic solutions of a particle in the potential $U(\phi)=-V(\phi)=\left(\mu^{2} / 2\right) \phi^{2}-(\lambda / 4) \phi^{4}$ (here with $\mu^{2}=2$ and $\left.\lambda=1\right)$ exist when the total energy of the particle lies between $E_{\max }=\frac{\mu^{4}}{4 \lambda}$ (top of the inverted potential) and $E_{\min }=0$. A particle with energy $E_{A}$ will undergo a periodic motion of period $P$, understood as the length of the extra-dimension. Note that this is precisely the potential used in our first example (12).

By inspection of Figure 1, whenever the inverted potential $U(\phi)$ has a global maximum not at $\phi=0$, there will always exist a range of values for the constant of integration $E_{A}$ such that at least one nontrivial solution, with appropriate boundary conditions, exists. Each solution $\phi_{A}(y)$ will actually be periodic, with period $T(A)$, and will reach its maximum value $A$ at $y=T / 4$. The constant parameter $E_{A}$ of each solution is set by the amplitude $A$ of the solution since $E_{A}=-V(A)$. Using (3), we may also derive an expression for the period $T$ as a function of $A$

$$
T(A)=2 \sqrt{2} \int_{0}^{A} \frac{d \phi}{\sqrt{V(\phi)-V(A)}} .
$$

It is straightforward to show that there is a family of possible nontrivial periodic solutions parametrized by the amplitude $A$. To relate the period $T(A)$ of a solution to the physical size of the interval $\pi R$ one simply notes 13 . that one has to be be a multiple of the other, i.e. $2 \pi R=$ $(m+1) T(A)$, where $m$ is a positive integer. In that case, the nontrivial solution will contain $m$ nodes inside the interval $[0, \pi R]$. As long as the constant $E_{A}$ has a value in the appropriate range, there will always be at least one nontrivial solution with $m=0$, i.e. nodeless in the orbifold interval. Depending on the function $T(A)$, there may be more than one value of $A$ such that $2 \pi R=T(A)$, and therefore more than one nontrivial nodeless solution.

On the other hand the function $T(A)$ must have a non-zero global minimum $T_{\min }$. Once $T_{\min }$ (determined by the specific potential $V(\phi)$ chosen) as well as the size of the orbifold interval $\pi R$ are known, then defining $2 \pi R / T_{\min }=\delta$, the maximum number of nodes that a nontrivial solution $\phi_{A}(y)$ can have is $m_{\max }=I P(\delta)-1$ (where $I P(x) \equiv \operatorname{IntegerPart}(x)$ gives the largest integer less than or equal to $x$.), and $m_{\max }+1$ is the maximum number of solution periods that can "fit" in $2 \pi R$.

This means that a finite number of nontrivial solutions with nodes are also possible.

The most important question about these background solutions concerns stability. To investigate this, we add small perturbations around one such solution $\phi_{A}(y)$ of (2), i.e. we write $\phi(x, y) \rightarrow \phi_{A}(y)+\varphi(x, y)$. Separation of variables $\varphi(x, y)=\varphi_{x}(x) \varphi_{y}(y)$ then yields

$$
\begin{aligned}
& \square \varphi_{x}^{n}(x)=-M_{n}^{2} \varphi_{x}^{n}(x) \\
& \varphi_{y}^{n^{\prime \prime}}(y)-q(y) \varphi_{y}^{n}(y)=-M_{n}^{2} \varphi_{y}^{n}(y),
\end{aligned}
$$

with

$$
q(y)=\left.\frac{\partial^{2} V}{\partial \phi^{2}}\right|_{\phi_{A}(y)},
$$

which are the equations of motion of a (Kaluza-Klein) tower of $4 \mathrm{D}$ scalar fields $\varphi_{x}^{n}(x)$ with masses $M_{n}^{2}$ and with profiles along the extra dimension given by $\varphi_{y}^{n}(y)$.

The masses $M_{n}^{2}$ and profiles $\varphi_{y}^{n}(y)$ are found by solving the second order linear differential equation (6).

Now, suppose we have identified a solution $\phi_{A}(y)$ of equation (2). Taking the derivative of (2), one obtains

$$
\phi_{A}^{\prime \prime \prime}(y)-q(y) \phi_{A}^{\prime}(y)=0 .
$$

Comparing Equations (8) and (6) we then see that the derivative $\phi_{A}^{\prime}(y)$ of the background solution actually corresponds to a massless mode $\left(M_{n}^{2}=0\right)$ of the perturbation $\varphi(x, y)$, although now with Neumann boundary conditions, rather than the Dirichlet ones we require ${ }^{1}$.

This is extremely useful, however, since the general theory of the eigenvalues of the Sturm-Liouville problem with Dirichlet (D), periodic (P), semiperiodic (S), and Neumann (N) boundary conditions implies the following chain of inequalities

$$
\begin{aligned}
\lambda_{0}^{N} & \leq \lambda_{0}^{P}<\lambda_{0}^{S} \leq\left\{\lambda_{0}^{D}, \lambda_{1}^{N}\right\} \leq \lambda_{1}^{S}<\lambda_{1}^{P} \leq\left\{\lambda_{1}^{D}, \lambda_{2}^{N}\right\} \\
& \leq \lambda_{2}^{P}<\lambda_{2}^{S} \leq\left\{\lambda_{2}^{D}, \lambda_{3}^{N}\right\} \leq \lambda_{3}^{S}<\lambda_{3}^{P} \leq\left\{\lambda_{3}^{D}, \lambda_{4}^{N}\right\} \\
& \leq \cdots
\end{aligned}
$$

relating the towers of eigenvalues corresponding to each different type of boundary condition.

Applying this to any solution $\phi_{A}(y)$ with greater than the minimal periodicity, we see that the associated derivative $\phi_{A}^{\prime}(y)$, obeying Neumann boundary conditions, will have multiple nodes in the interval $[0, \pi R]$.

\footnotetext{
1 This should not come as a big surprise, since it is just the translation mode, the masslessness of which is a reflection of translation symmetry. It cannot be a physical solution since translation invariance is broken in the orbifold.
} 
Thus we may identify it as the eigensolution $\varphi_{i}^{N}(y)$, with $i \geq 2$, with its masslessness implying that the corresponding eigenvalue obeys $\lambda_{i}^{N}=0$.

However (9) implies that $\lambda_{2}^{N}>\lambda_{0}^{D}$. Therefore, if $\lambda_{i}^{N}=$ 0 for some $i \geq 2$, then there exists at least one $\left(\lambda_{0}^{D}\right)$ eigenvalue of the Dirichlet problem, and possibly more, that are negative!

Thus, all static solutions with nodes in the interval are unstable.

What remain are the lowest-lying solutions, with no nodes in $(0, \pi R)$. Note again that, depending on the complexity of the potential, there may be multiple static, nodeless solutions with the same periodicity. A different approach is necessary to investigate their stability, and here we shall merely state the result. It turns out that the key ingredient is the dependence of the periodicity of static solutions $\mathrm{T}$ on their amplitude $A$.

It is possible to prove the following general result. $A$ static, nodeless solution $\phi_{A_{*}}(y)$ to equation (2), with amplitude $A_{*}$, and period $T\left(A_{*}\right)$, and satisfying $\phi_{A_{*}}(0)=$ $\phi_{A_{*}}(T / 2)=0$, is stable if and only if

$$
\left.\frac{d T}{d A}\right|_{A=A_{*}}>0
$$

This is the central result presented in this letter, and we reserve the somewhat involved proof for our companion paper [1].

Further, since the energy of such a solution is given by

$$
E(A)=V(A) T(A)+4 \sqrt{2} \int_{0}^{A} \sqrt{V(\phi)-V(A)} d \phi
$$

when there are multiple metastable nodeless solutions with the same value of $T$, it is straightforward to compare their energies and determine which is the lowest lying state.

After all this generality, it may prove useful to sketch how some of this works out in some concrete examples.

Let us first consider an exactly solvable example defined by the potential

$$
V(\phi)=-\frac{\mu^{2}}{2} \phi^{2}+\frac{\lambda}{4} \phi^{4}
$$

where $[\mu]=[\lambda]^{-1}=$ (Mass).

It is easy to see that for $E=\frac{\mu^{4}}{4 \lambda}$ one obtains non-trivial solutions of Eq. (3) known as the kink and anti-kink

$$
\phi_{(\operatorname{anti}-) \text { kink }}(y)= \pm \frac{\mu}{\sqrt{\lambda}} \tanh \left[\frac{\mu}{\sqrt{2}}\left(y-y_{o}\right)\right]
$$

where the kink location $y_{o}$ should be set to zero because of the boundary conditions of the scalar field. This solution interpolates along the (now infinite) extra dimension between the constant background solutions $\phi_{ \pm} \equiv \pm \mu / \sqrt{\lambda}$.
For $0<E<\frac{\mu^{4}}{4 \lambda}$, we can still integrate Eq. (3) to obtain [13]

$$
\phi_{k}(y)= \pm \frac{\mu}{\sqrt{\lambda}} \sqrt{\frac{2 k^{2}}{k^{2}+1}} \operatorname{sn}\left(\frac{\mu}{\sqrt{k^{2}+1}} y, k^{2}\right),
$$

where

$$
k^{2}=\frac{\mu^{2}-\sqrt{\mu^{4}-4 \lambda E}}{\mu^{2}+\sqrt{\mu^{4}-4 \lambda E}}
$$

and $\operatorname{sn}\left(x, k^{2}\right)$ is the Jacobi Elliptic Sine-Amplitude, parametrized by the elliptic modulus $k$ (a real parameter such that $0<k<1$ ) and with period $4 K$, where

$$
K\left(k^{2}\right)=\int_{0}^{\pi / 2} \frac{d \theta}{\sqrt{1-k^{2} \sin ^{2} \theta}}
$$

is the complete elliptic integral of the first kind.

In this example, it is possible to show that the total number $n_{\max }$ of nontrivial solutions is given by $n_{\max }=$ $\operatorname{IP}(\mu R)-1$. Since $\mu$ is a fixed parameter of the scalar potential and $R$ is the fixed radius of the extra dimension, $n_{\max }$ is completely specified by the model.

The complete set of static nontrivial background solutions consistent with the boundary conditions, for the potential (12) is then

$$
\phi_{k_{n}}(y)= \pm \frac{\mu}{\sqrt{\lambda}} \sqrt{\frac{2 k_{n}^{2}}{k_{n}^{2}+1}} \text { sn }\left(\frac{\mu}{\sqrt{k_{n}^{2}+1}} y, k_{n}^{2}\right),
$$

where $n$ is an integer such that $0 \leq n \leq n_{\max }$.

The radius $R$ of the extra dimension is such that $2 \pi R=$ $\frac{4}{\mu} \sqrt{k_{0}+1} K\left(k_{0}^{2}\right)$.

The solution with lowest energy, and no nodes in the interval, will be $\phi_{k_{0}}(y)$ (using $k$ as an equivalent label to $A$ ) and is plotted in Fig. (2). The rest of solutions $\phi_{k_{n}}(y)$ will have nodes and increasing energy. And thanks to our general stability argument they will be unstable.

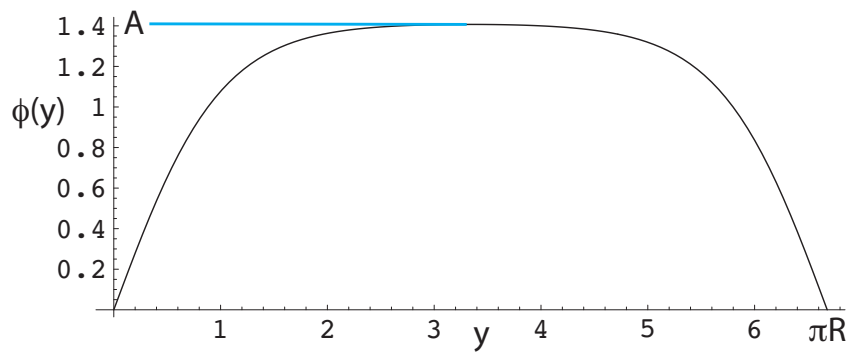

FIG. 2: The single stable kink solution for the case of the potential of our first example (12).

A second example, useful for visualizing the possibility of multiple stable solutions, is provided by the (admittedly contrived) potential

$$
V(\phi)=-\phi^{2}-5 \phi^{4}+\frac{5}{2} \phi^{6}-\frac{1}{3} \phi^{8}+\frac{1}{77} \phi^{10},
$$


in which we have set all dimensionful parameters to unity.

Obviously, our general considerations imply that the only possible stable configurations are the nodeless ones. In figure 3 we plot the inverted potential $U(\phi)$ for $\phi>0$ and the period $T(A)$ of static solutions with amplitude A.

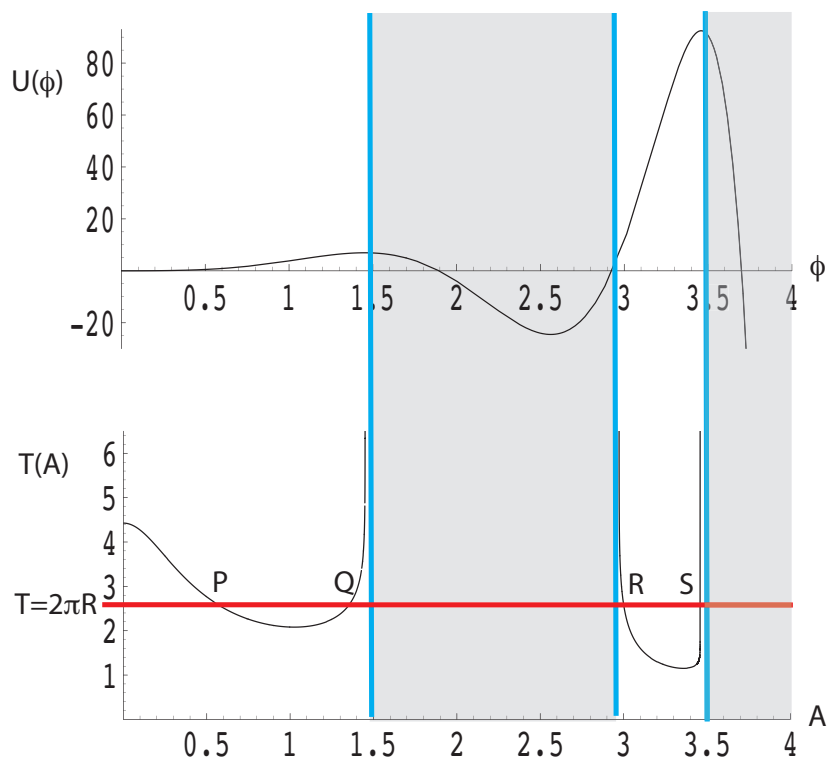

FIG. 3: For this example of the potential, there exist four distinct nodeless solutions, here labeled as $P, Q, R$ and $S$, with different values of the amplitude $A$, but with the same period. Those at $P$ and $R$ are unstable, while those at $Q$ and $S$ are stable. Further, (11) implies that $S$ is of lower energy than $Q$. In the shaded regions there are no solutions with the appropriate boundary conditions.

If we are looking for nodeless solutions, we are interested in those values of $A$ satisfying $T(A)=2 \pi R$ and by inspection there are four such solutions, labeled $P, Q$, $R$ and $S$. Furthermore, our stability criterion (10) then implies that $P$ and $R$ are unstable, whereas $Q$ and $S$ are distinct, stable, nodeless solutions. These two solutions are plotted in Fig. (4)

In addition, using (11), one can show that the solution denoted by $S$ is the lowest energy one.

In this letter we have investigated the existence of a finite tower of nontrivial static configurations in orbifolded extra dimension models containing bulk scalar fields with potentials with multiple degenerate vacua. We have described a proof that all but the lowest-lying of these configurations are classically unstable, and have discussed a general stability criterion for the lowest, nodeless, modes. We have then illustrated these general results first with the case of an exactly solvable model and then with a more complicated but also richer example.

We have thus analyzed an entirely new class of metastable bulk field configurations around which novel particle physics and cosmological phenomena may occur.

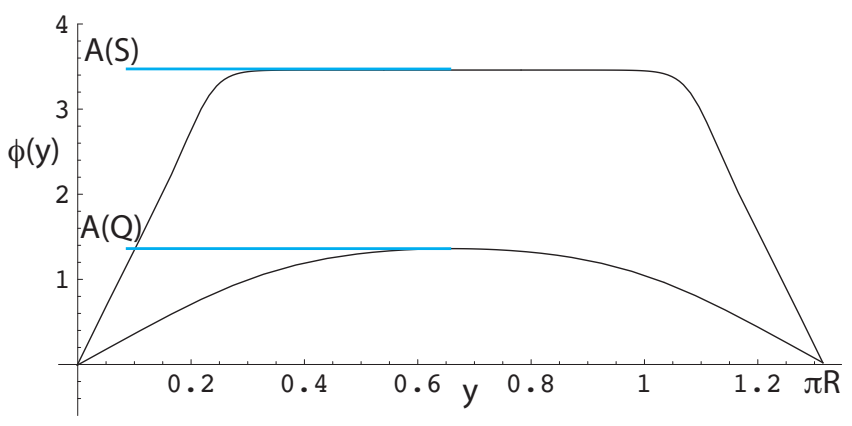

FIG. 4: The two stable solutions (points $Q$ and $S$ in figure (3) for the potential (18). The solution at point $S$, with the larger amplitude, $A(S)$, has the lower energy.

The stability criterion (10) of the lowest lying modes requires an involved proof, which we reserve for our companion paper, in which we also present a detailed discussion of the configurations described in this letter. We also apply these considerations to a range of models.

Natural further steps are the inclusion of gravity into the analysis, and a comprehensive investigation of the applications of our results. These studies are already underway.

We thank Tim Tait and James Wells for discussions. M.Toharia is supported by funds provided by Syracuse University and the U.S. DOE under Contract number. DE-FG-02-85ER 40231. M. Trodden is supported by the National Science Foundation under grant PHY-0354990 and by Research Corporation.

[1] M. Toharia and M. Trodden, arXiv:0708.4008 [hep-ph].

[2] V. A. Rubakov and M. E. Shaposhnikov, Phys. Lett. B 125 (1983) 136.

[3] K. Akama, Lect. Notes Phys. 176, 267 (1982) arXiv:hep-th/0001113.

[4] I. Antoniadis, Phys. Lett. B 246, 377 (1990).

[5] J. D. Lykken, Phys. Rev. D 54, 3693 (1996) arXiv:hep-th/9603133.

[6] N. Arkani-Hamed, S. Dimopoulos and G. R. Dvali, Phys. Lett. B 429, 263 (1998) arXiv:hep-ph/9803315.

[7] I. Antoniadis, N. Arkani-Hamed, S. Dimopoulos and G. R. Dvali, Phys. Lett. B 436, 257 (1998) arXiv:hep-ph/9804398.

[8] L. Randall and R. Sundrum, Phys. Rev. Lett. 83 (1999) 3370 arXiv:hep-ph/9905221.

[9] L. Randall and R. Sundrum, Phys. Rev. Lett. 83, 4690 (1999) arXiv:hep-th/9906064.

[10] N. Arkani-Hamed and M. Schmaltz, Phys. Rev. D 61, 033005 (2000) arXiv:hep-ph/9903417.

[11] H. Georgi, A. K. Grant and G. Hailu, Phys. Rev. D 63, 064027 (2001) arXiv:hep-ph/0007350,

[12] D. E. Kaplan and T. M. Tait, JHEP 0111, 051 (2001) arXiv:hep-ph/0110126.

[13] B. Grzadkowski and M. Toharia, Nucl. Phys. B 686, 165 (2004) arXiv:hep-ph/0401108. 